\title{
Importancia de los Semilleros De INVESTIGACIÓN EN LA UNIVERSIDAD de Cartagena
}

\author{
Por: Jorge Llamas Chávez
}

\section{RESUMEN}

El propósito de este documento es generar unas reflexiones entorno al fenómeno de los semilleros de investigación en la Universidad de Cartagena, referente a su dinámica, planteándose la necesidad de articular el trabajo investigativo a la formación, el cual compromete a docentes, estudiantes y centros de investigación a generar reflexiones sobre las nuevas formas de enseñar la investigación. En ese sentido, las ideas expuestas hacen referencia a las características de los semilleros. Esta caracterización se hace con base a un estudio que se viene adelantando con el apoyo financiero del Centro de Investigaciones que recoge en gran parte la dinámica investigativa de estos semilleros en la Universidad.

\section{Constitución de Semilleros}

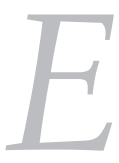

n Colombia, el tema de la investigación a nivel de pregrado no se tenia en cuenta como estrategia para la formación profesional, pues siempre se miraba como una tradición correspondiente a estudios de postgrado, maestría o doctorados. A medida que la universidad fue actualizando su sistema de investigación, surgieron estrategias extracurriculares que reafirmaban la necesidad de vincular estudiantes de los diferentes programas de pregrado al quehacer investigativo, convirtiéndose poco a poco en un soporte del trabajo de los investigadores y grupos de investigación. En ese sentido, nos invita a pensar en la necesidad urgente de articular el trabajo investigativo a la formación.

Por fuera del desarrollo curricular, los estudiantes por sí solos o en compañía de docentes, han creado espacios alternos de reflexión y análisis alrededor de la actividad investigativa. La curiosidad, la pregunta, la discusión, el trabajo en grupo y la motivación por ser parte de una comunidad científica en pro del desarrollo, se constituyeron en factores determinantes para que muchos estudiantes se organizaran alrededor de lo que se llama los Semilleros de Investigación, que continuamente incentivan la actividad investigativa, desde el ser y el hacer dentro de la universidad. 
El termino "semillero" significa "sitio donde se siembra para luego transplantar". ${ }^{1}$ Es decir, es el lugar o espacio que ofrece las condiciones iniciales para su germinación. Desde esta perspectiva, es nuestra labor generar esas condiciones para que la semilla -futuros investigadores- constituya a su tiempo ese caudal de profesionales que desde la investigación den respuesta a la complejidad de

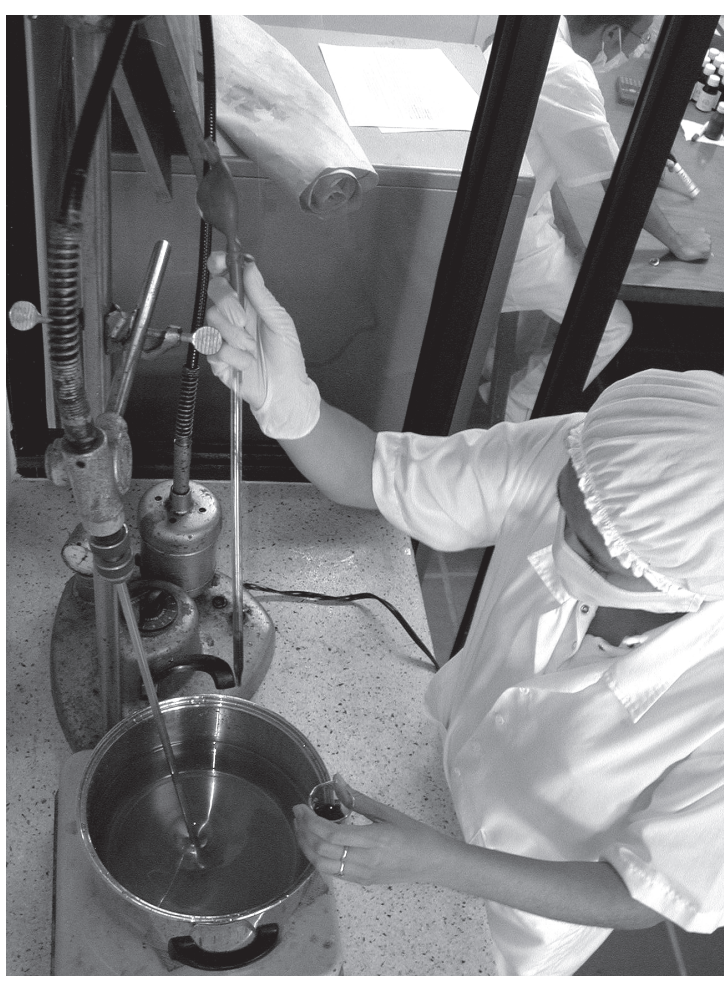

Foto: Mario Lorduy. Sección Publicaciones. Universidad de Cartagena. problemas de nuestra sociedad. Esas condiciones incluyen la superación de prejuicios y la dotación en el uso eficiente del avance científico y tecnológico, que conlleven a la aceptación del trabajo interdisciplinario donde se puedan integrar los saberes que permitan generar condiciones reales para el crecimiento de ésta.

Existen muchas definiciones de Semilleros de Investigación, entre las cuales me permito resaltar las siguientes:

"Espacio crítico que permite a aquellos profesionales reflexionar sobre sus procesos formativos, reconocer falencias y explorar procesos que potencien la creatividad y la capacidad de gestión propios de las generaciones a formar". ${ }^{2}$

"Espacio donde los docentes y estudiantes tendrán oportunidad de reflexionar y construir conocimientos y vincularse en propuestas que propicien nuevos

${ }^{1}$ Diccionario Larousse (2003), Editorial Norma, Pag. 590, Bogotá D.C., Colombia.

2 Red - SIN. Red de Semilleros de Investigación de la Universidad de Antioquia ( 2001 ), Fondo Editorial Biogénesis, Primera Edición, Medellín, Colombia.

${ }^{3}$ POLITÉCNICO JAIME CADAVID, Documento Semillero de Investigación (sin otros datos).

${ }^{4}$ Universidad Nacional, Publicación Semilleros de Investigación ( $\sin$ otros datos). avances en la ciencia y la tecnología, en pro del desarrollo social, con una visión prospectiva en busca de la consolidación de un grupo de investigación de alto nivel". ${ }^{3}$

"Es el escenario que busca integrar el proceso de formación de los talentos humanos, procesos llenos de aprendizajes y olvidos, de acuerdos y tensiones, de convergencias y divergencias, de universidades y particularidades. Permite la integración de profesores de reciente vinculación con profesores experimentados y de amplios conocimientos siguiendo una dinámica que enriquece la construcción del quehacer académico". ${ }^{4}$

Nuestra institución también ha hecho su propia definición de Semilleros de Investigación, basada en sus experiencias de la practica investigativa de los estudiantes, expresada en el acuerdo No. 09 BIS del Consejo Superior de la Universidad de Cartagena del 26 de mayo de 2003, en los siguientes términos: 
"Espacios Académicos orientados a la formación para la practica investigativa y la consolidación de un cultura investigativa en los estudios de pregrado y post - grado, que contribuyan a la formación de proyectos que generen, adopten y transfieren conocimientos para la solución de problemas de la realidad social, política, cultural, científica, tecnológica y económica de la región y el país". 5

De esta manera los semilleros de investigación fundan su importancia en que buscan sensibilizar a los estudiantes frente a una realidad, orientándoles hacia las soluciones de los diferentes problemas que están afectando nuestra región, país o comunidad y abriendo espacios donde se den procesos investigativos orientados a una formación integral, que den como resultado la generación de conocimientos con las cuales se pueda transformar nuestra realidad actual.

La Universidad de Cartagena se ha caracterizado por su liderazgo regional en el campo investigativo en diferentes áreas del conocimiento, contando con grupos de investigación reconocidos por COLCIENCIAS. Las experiencias de formación en el pregrado no se quedan atrás y empiezan a mostrar sus productos; entre éstos, los Semilleros de Investigación, los Jóvenes Investigadores y la iniciativa de constituir la Red de Semilleros de Investigación que se comenzó a gestar a partir del

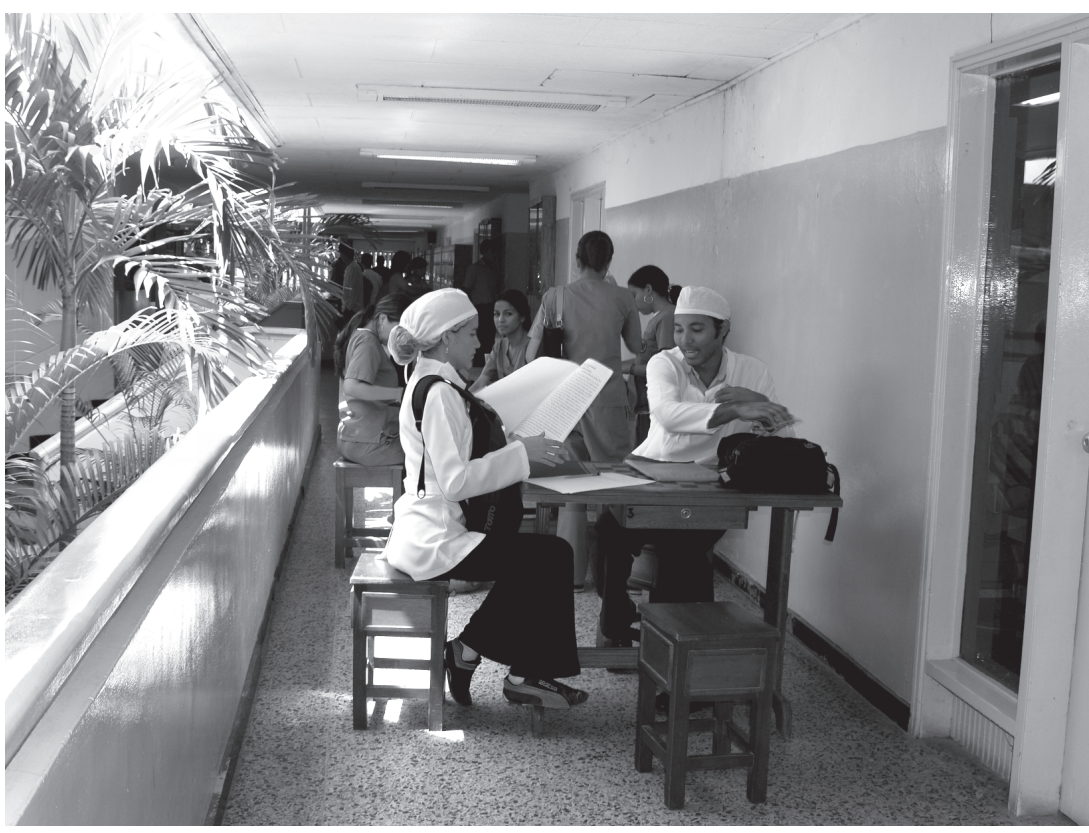

Foto: Mario Lorduy. Sección Publicaciones. Universidad de Cartagena. primer encuentro en esta modalidad, realizado el 11 y 12 de mayo de 2006, con el objeto de agrupar las diferentes experiencias de formación que se desarrollan al interior de la Universidad de Cartagena.

${ }^{5}$ ACUERDO 09 - Bis, del Consejo Superior de la Unversidad de Cartagena, "Por el cual se reglamenta los grupos de semilleros de investigación de estudiantes de pregrado y postgrado. 26 de mayo de 2006. 
- Semillero en Catálisis y Nuevos Materiales. SEMILICATUC. Programa de Química y Farmacia.

- Semillero de Bioinformática. Programa de Química y Farmacia.

- Semillero Carboquímica. Programa de Química.

- Semillero JOINEN. Facultad de Enfermería.

- Semillero Administración de la Salud. Programa a Distancia (en proceso de formación).

- Semillero Amenazas Naturales GIAN. Programa de Ingeniería Civil.

- Semillero Proteínas Alimentarias. Programa de Ingeniería de Alimento.

- Semillero Escuela del Programa de Ingeniería Química. SEPIQ. Programa de Ingeniería Química.

- Semillero Instituto de Hidráulica y Saneamiento Ambiental ISHA. Programa de Ingeniería Civil.

- Semillero Ciencia, Tecnología, Sociedad CTS. Interfacultad. Ciencias Sociales y Educación, Ciencias Económicas y Ciencias e Ingeniería.

- Semillero Filosofía del Derecho. Facultad de Derecho

- Semillero Historia de las Ideas Políticas. Facultad de Derecho

- Semillero Sociedad, Raza y Poder. Instituto Internacional del Caribe.

- Semillero Desarrollo Local y Gestión Publica. Interdisciplinario (Facultad de Ciencias Sociales y Educación - Instituto Internacional de Estudios del Caribe.

- Semillero SIFACSE. Facultad de Ciencias Sociales y Educación.

- Semillero Estudios Históricos y Filosóficos. Programa de Historia.

- Semillero Mercado Laboral. Programa de Economía.

- Semillero Alergia Experimental. Instituto de Inmunologia. ${ }^{6}$

En la perspectiva anterior, la Universidad de Cartagena ha abierto los espacios para que se consolide la cultura investigativa a través de los semilleros de investigación, iniciativa que esta en concordancia con las políticas nacionales de fomento de la investigación emanadas de COLCIENCIAS , a partir de procesos sustentados en la metodología "aprender haciendo" y con los objetivos institucionales de la Universidad. Se trata por tanto, de articular los esfuerzos dispersos que se vienen dando entre los diferentes grupos de investigación y unidades académicas de la Universidad para consolidar así la iniciativa de Semilleros de Investigación como estrategia de formación investigativa.

${ }^{6} \mathrm{Est} r$ a te g i a s Productivas para el Fomento de Semilleros de Investigación en la Universidad de C a r t a g e $\mathrm{n} \mathrm{a}$. Investigación que actualmente adelanta el grupo CTS, aprobada por el CICTE, 2006.
Entonces, le corresponde a la Universidad no solamente la formación para el trabajo en el caso de los profesionales sino la formación de los científicos que han de innovar saberes o adaptar tecnologías. Así, la Universidad se ubica en el centro del desarrollo científico y tecnológico y de las políticas que buscan acelerarlo, de producir saberes es la tarea al lado de institutos gubernamentales, centros de investigación, comunidades académicas y otros. 
En todo caso, la actividad científica técnica en la Universidad de Cartagena ha ido construyendo su propia cultura y formando líneas de investigación que regulan la practica investigativa permitiendo generar en cada una de las etapas de pregrado y postgrado una iniciativa de interés por desarrollar y consolidar una formación científica . Por esta razón, los Semilleros de Investigación hacen parte del contexto de la investigación y están a su vez determinados por un proceso de formación: de ahí su importancia y sus correlaciones.

\section{BIBLIOGRAFIA}

ACUERDO No. 09 - Bis, del Consejo Superior de la Universidad de Cartagena. (2006) "Por el cual se reglamenta los grupos de semilleros de investigación de estudiantes de pregrado y postgrado. Universidad de Cartagena

DICCIONARIO LAROUSSE. (2003) Editorial Norma, pag. 590, Bogota D.C. Colombia

DOCUMENTO DE TRABAJO. (2006) Primer Encuentro de Semilleros de Investigación, Universidad de Cartagena, Grupo de Investigación CTS, Universidad de Cartagena.

File://C:Convocatorias.htm.Vicerrectoria de Investigación. (2006) Universidad de Antioquia.

GÓMEZ BUENDÍA, Hernando. (1998) Educación: la agenda del siglo XXI hacia un desarrollo Humano. Editores Tercer Mundo, Primera Edición.

POLITÉCNICO JAIME CADAVID. Documento Semilleros de Investigación (sin otros datos)

RED SIN. (2005) Red de Semilleros de Investigación de la Universidad de Antioquia. Fondo Editorial Biogénesis, Primera Edición, Medellín Colombia.

RESTREPO, Bernardo. Conceptos y Aplicaciones de la Investigación Formativa, y Criterios para evaluar la Investigación Científica en Sentido Estricto, pag. 3 - 6 (sin otros datos)

TORREGROZA, Edilbert. (2003) Modulo de Sistema Nacional de Ciencia y Tecnología. Universidad de Cartagena.

UNIVERSIDAD DE CARTAGENA. (2006) Estrategias Productivas para el Fomento de Semilleros de Investigación en la Universidad de Cartagena. Grupo de Investigación CTS., Proyecto de Investigación en ejecución.

UNIVERSIDAD NACIONAL - Publicación Semilleros de Investigación.(sin otros datos)

BIOGRAFIA

JORGE LLAMAS CHAVEZ

Trabajador Social, Universidad de Cartagena. Especialista en Teorías, Métodos y Técnicas de Investigación Social, Universidad de Cartagena. Miembro del Grupo de Investigación (COLCIENCIAS) Ciencia, Tecnología y Sociedad (C.T.S.). Docente Facultad de Ciencias Sociales y Educación. 